\title{
Uniocular Pulfrich phenomenon: an abnormality of visual perception
}

\author{
J. J. ELL AND M. A. GRESTY
}

From the Medical Research Council, Neuro-Otology Unit (formerly Hearing and Balance Unit), Institute of Neurology, National Hospital, Queen Square, London WC1N 3BG

SUMmaRY We describe a patient with multiple sclerosis who experienced the Pulfrich illusion of elliptical motion of a target moving linearly when viewing the motion with one eye as opposed to the well recognised binocular manifestation of the phenomenon. The perception of the illusion was independent of the wave form or velocity characteristics of target motion or of retinal image position. We suggest that the occurrence of the phenomenon does not simply reflect delay in the visual system but is a function of an abnormality of perceptual interpretation of visual stimuli occurring at a high integrative level.

In 1922 C. von Pulfrich demonstrated that if a filter is held in front of one eye in a normally sighted individual a pendulum bob swinging in the frontal plane is perceived as moving with an elliptical trajectory. ${ }^{1}$ His explanation of the phenomenon that has come to bear his name was that there was a prolongation of latency of vision in the dimmed eye. His theory was supported by the work of Julesz and White, ${ }^{2}$ who extended the model of a 'delay-line' hypothesis to incorporate a short-term visual memory system with separate mechanisms for stereopsis and binocular combination, each having a different time constant. Frisen et al. ${ }^{3}$ drew attention to the occurrence of the phenomenon in patients with minor optic nerve involvement from multiple sclerosis. We should like to report a case of an illusion with similar characteristics occurring with monocular vision and the results of some examinations.

\section{Case report and investigations}

A 29-year-old woman with definite multiple sclerosis and a positive family history of this disease presented initially 5 years ago with an episode of right retrobulbar neuritis. She had a further attack one year later. After recovery from the first episode she noted that, when she looked with the right eye alone, the trajectory of moving objects appeared distorted. When she viewed moving objects binocularly, the illusion was absent.

At the time of our examination there was temporal Correspondence to Dr J. J. Ell. pallor of the right optic disc. Visual acuity on the right was $6 / 12$ correcting to $6 / 4$ with a pinhole, on the left $6 / 4$. She saw 13 out of 14 of the Ishihara plates correctly but reported a subjective $50 \%$ desaturation to red with the right eye. Visual evoked responses in 1978 had shown a delayed latency on the right at 139 ms and a normal latency on the left at $98 \mathrm{~ms}$. Halffield stimulation showed no additional disparity of latencies. The patient was a very accurate and reliable witness.

The behavioural characteristics of her monocular visual illusion were examined with a $5 \mathrm{~mm}$ diameter red target generated by a helium-neon laser reflected on to a screen at $2 \mathrm{~m}$ from the patient. The spot could be made to move in the horizontal or vertical plane with either a sinusoidal or ramp wave form subtending an angle which was variable up to a maximum of $44^{\circ}$ of arc. The patient viewed the target both in darkness and with ambient illumination.

The target was made to oscillate at frequencies 0.002 to $10 \mathrm{~Hz}$. The perceived trajectories at different frequencies are outlined in Fig. 1. The limiting frequency for the perception of motion which also produced autokinesis of the target was the same for each eye at $0.008 \mathrm{~Hz}$. At $0.02 \mathrm{~Hz}$ the target, when viewed with the right (affected) eye, appeared to move in a half ellipse. Between $0.05 \mathrm{~Hz}$ and $5 \mathrm{~Hz}$ there was an illusion of elliptical motion. Above $5 \mathrm{~Hz}$ the right eye perceived linear motion, and at these higher frequencies the target had the appearance of a continuous line. When the target was moving fror left to right it appeared to be approaching anc 


$\begin{array}{lll}\begin{array}{l}\text { Target Frequency } \mathrm{Hz} \\ \text { (ramp or sinusoidal) }\end{array} & \begin{array}{c}\text { Perceived Trajectory } \\ \text { left eye }\end{array} & \text { Comments } \\ 0.002 & \text { autokinesis of target no perception of linear motion } \\ \text { to } & & \end{array}$

0.01

to

0.02

0.05

to

1.0

2.0

to

5.0

plus
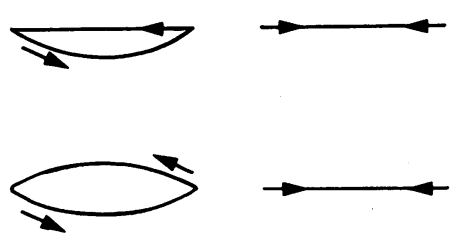

constant ratio of length to depth of illusion

depth of illusion shrinks
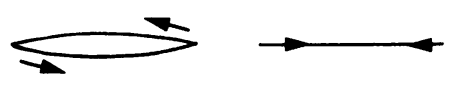

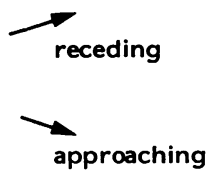

enlarging; when moving right to left it appeared to recede and diminish in size. This directionality was found to alter from one test session to another. Targets moving unidirectionally always appeared to be approaching. Between 1 and $5 \mathrm{~Hz}$ the perceived 'width' of the ellipse diminished from $10 \mathrm{~cm}$ until a straight line was seen. The characteristics of the illusion were the same whether a sinusoidal or ramp wave form was presented and were identical both in darkness and with ambient illumination. The illusion occurred equally whether the patient attempted to track the target or maintained the eye in the primary position. The illusion as described was the same for target motion in the vertical plane. Objects truly receding and approaching were perceived normally. A target moving at $2 \mathrm{~Hz}$ with a saw-tooth wave form having a velocity asymmetry of 10:1 induced a very strong sense of ego motion in the direction of the fast phase. This was not experienced with the target moving at $1 \mathrm{~Hz}$, nor was ego motion experienced at either frequency by normal observers.

\section{Discussion}

To our knowledge the occurrence of the Pulfrich phenomenon with monocular vision has not previously been reported. Its occurrence and characteristics as outlined raise important points concerning the explanation of the visual illusion. In the case of a normal subject with a filter before one eye the binocular disparity is proportional to the velocity of the target. In the case of a target moving in simple harmonic motion an elliptical trajectory is perceived. However, in our case presentation of a target moving at constant velocity did not alter the shape of the perceived ellipse. It is known that the perceptual latencies for foveal and extra-foveal stimuli are different. ${ }^{4}$ Delay in visual perception ${ }^{5}$ and in visual evoked responses (VER) ${ }^{6}$ following retrobulbar neuritis has been clearly demonstrated. An alternative explanation of Pulfrich's phenomenon based on saccadic suppression has been put forward. ${ }^{7}$ The fact that our patient noted no difference in the 
illusion while tracking the target as opposed to viewing it with the eye still is evidence against either the saccadic suppression theory or the explanation of differential delay between perception of stimuli perceived from the peripheral or central fields.

In his demonstration of the use of the Pulfrich pendulum as a method for detection of abnormal delay in the visual pathway of patients with suspected multiple sclerosis Rushton found a strong correlation between abnormality on the pendulum test and delay of the VER. ${ }^{8}$ However, he was surprised to find no significant correlation between the size and direction of the interocular latency difference obtained from the Pulfrich pendulum and that obtained from the VER. As a possible explanation he suggested that small tracking variations may place the image on areas of retina of widely varying latency. This could be a possible explanation for the monocular Pulfrich phenomienon. Once again the fact that in our patient the illusion was not altered by foveal viewing as opposed to combined foveal and peripheral retinal stimulation is somewhat against this hypothesis. Rushton thought that cortical mediated mechanisms of depth perception were not implicated in the phenomenon, and our patient's observation that the presence or absence of ambient illumination made no difference supports his view in that she was not influenced by depth cues.

In view of the frequent occurrence of unilateral optic neuritis with corresponding delay in the VER one might ask why this phenomenon is comparatively rare. Furthermore the basic question arises: at what level in the visual system does the 'delay' take place? Observations to date would suggest that the Pulfrich phenomenon is due to an alteration in visual perception and thereby mediated primarily by the visual cortex. In their discussion Julesż and White postulated separate mechanisms for stereopsis (the localisation of binocular information in depth) and the cyclopean view (the binocular combination of the 2 eyes' views into a single percept). Each system could be thought of as having its own time constant for integration of successive images. For the case of the Pulfrich phenomenon on binocular vision they postulated a short-term visual memory with 2 different time constants. None of the current theories can explain the occurrence of the Pulfrich phenomenon with monocular vision. In our case the single requirement of the visual stimulus was that the subject could perceive target motion; depth cues, direction and velocity of motion, and retinotopic cortical localisation were unimportant.

Zeki' has established that the posterior bank of the superior temporal sulcus of the rhesus monkey contains neurones which signal motion of visual targets towards or away from the animal. These are termed opposed movement complex and hypercomplex cells. The source of cues to the directionality of target motion has to be binocular in the case of discrete point targets, for the signalling of direction relies on opposed directions of target motion movement on the 2 retinae. The directionality of a large target may be detected monocularly through displacement in opposed directions of the retinal images of various parts of the target. In the case of our patient a small target viewed monocularly was seen to approach or recede, although logically the perception of such motion in depth requires binocular input. These considerations afford a possible explanation of the monocular Pulfrich illusion, namely, that at a cortical level concerned with motion in depth there is a faulty processing of the visual signals which mimics the situation of a suitably opposed signal arising from the occluded eye. As such the phenomenon is analogous to synchiria.

It is evident that the illusion of motion in 3 dimensions experienced by our patient while viewing an object moving in a linear trajectory is not attributable to functional derangement of the retinocortical projections and in particular is not a simple function of delayed conduction velocity. The reasons for this, reiterated, are that the shape of the trajectory of perceived motion was independent of the characteristics of the real motion of the target; in addition the illusion was not altered by foveal as opposed to combined peripheral and foveal viewing, the latter excluding an explanation involving different conduction velocities from different parts of the retina. That the illusion of elliptical motion in the case of our patient was dependent solely on the perception that something was moving and was otherwise unrelated to the physical parameters of motion suggests that the illusion reflects an abnormality of perceptual interpretation of visual stimuli occurring at a high integrative level.

We thank Dr P. Gautier-Smith for his kind permission to report this case.

\section{References}

1 Pulfrich $\mathrm{C}$ von. Die Stereoskopie im Dienste der Isochromen und Heterochromen Photometrie. Naturwissenschaften 1922; 10: 553-64. 569-74. 596-601. 714-22, 735-43, 751-61.

2 Julesz B. White B. Short term visual memory and the Pulfrich phenomenon. Nature 1969: 222: 639-41.

3 Frisen L. Hoyt WR. Bird AC. Weale RA. Diagnostic uses of the Pulfrich phenomenon. Lancet 1973; ii: 385-6.

4 Payne WH. Reaction time as a function of retinal reaction. Vision Res 1966: 6: 729-32.

5 Heron JR. Regan D. Milner BA. Delay in visual perception in unilateral optic atrophy after retrobulbar neuritis. Brain 1974; 97: 69-78. 
6 Halliday AM. McDonald WI. Mushin J. Delayed visual evoked response in optic neuritis. Lancet 1972; i: 982-5.

7 Harker GS. A saccadic suppression explanation of the Pulfrich phenomenon. Perception and Psychophysics 1967; 2: 423-6.

8 Rushton D. Use of the Pulfrich pendulum for detecting abnormal delay in the visual pathway in multiple sclerosis. Brain 1975; 98: 283-96.

9 Zeki SM. Cells responding to changing image size and disparity in the cortex of the rhesus monkey. J Physiol 1974; 242: 827-41. 